\title{
Botulinomtoxin als effektives Therapeutikum bei Inkontinenz
}

- Botulinumtoxin (z.B. Botox ${ }^{\oplus}$ ) ist bislang als Schönheitsmittel bekannt. Doch auch im Kampf gegen die Inkontinenz ist Botulinumtoxin mittlerweile ein probates Therapeutikum mit Langzeitwirkung. Prof. Klaus-Peter Jünemann, Vorsitzender der Deutschen Kontinenz Gesellschaft und Direktor der Klinik für Urologie und Kinderurologie an der Universitätsklinik in Kiel meint:„Ich halte Botox ${ }^{\circledast}$ im urologischen Bereich für das innovativste pharmazeutische Produkt seit Viagra ${ }^{\circledR}$."

Bisher erhielten Patienten, die aufgrund einer instabilen Blase unter Inkontinenz litten, oft eine medikamentöse Therapie. Doch diese hat mitunter starke Nebenwirkungen, bei- spielsweise ein schwindendes Kurzzeitgedächtnis. Auch die erneut auftretende Blasenschwäche bei nachlassender Wirkung der Medikamente befreiten die Patienten nicht aus ihrer Isolation. Einzige Alternative waren operative Eingriffe.

Die neue Behandlungsmöglichkeit hingegen lindert die Leiden vieler Inkontinenzpatienten und erspart ihnen invasive Therapien. Hierbei wird etwa einmal im Jahr Botulinumtoxin direkt in die Blase gespritzt. Jünemann verweist auf eine amerikanischen Studie von Dezember 2010, die zeigt, dass Patienten, deren medikamentöse Behandlung nicht erfolgreich war, mit hervorragendem Langzeiteffekt mit Bo- tulinumtoxin behandelt werden können. Das bedeutet eine Steigerung der Lebensqualität für viele Menschen.

„Mittlerweile haben wir hier, an der Universitätsklinik Kiel, viel Erfahrung mit der Botulinumtoxin-Therapie. Wir behandeln rund 200 Patienten im Jahr. Unsere Klientel reicht von Männern mit verschiedenen Prostataerkrankungen bis zu Frauen mit Blasensenkung und Dranginkontinenz." berichtet Jünemann. Weitere Informationen sind erhältlich unter www.kontinenz-gesellschaft.de.

Nach Informationen der

Deutschen Kontinenz Gesellschaft, Kassel

\section{Blasen- und Nierentee - Helfer bei Harnwegsinfekten}

— Heilpflanzentees können zum einen Harnwegsinfekten vorbeugen und zum anderen eine Antibiotikatherapie bei akuter Blasenentzündung begleiten. Denn in solchen Fällen ist es wichtig, die Bakterien auszuspülen. Mindestens zwei Liter Flüssigkeit am Tag sollten Patienten, die unter einer Blasenentzündung leiden, zu sich nehmen - auch wenn bereits ein Antibiotikum gegen die Entzündung verordnet wurde. Mit einer sogenannten Durchspülungstherapie (z. B. mit Sidroga ${ }^{\circledR}$ Blasen- und Nierentee oder Sidroga ${ }^{\circledR}$ Brennnesselblätter) wird der Harn verdünnt und die Harnmenge erhöht. Der Sidroga ${ }^{\circledast}$ Blasen- und Nierentee setzt zu diesem Zweck auf eine Kombination aus Birken-, und Orthosiphonblättern (Flavonoide), Riesengoldrutenkraut (Flavonoide, Saponine) und Hauhechelwurzel (Isoflavonoide). Der regelmäßige Genuss des Tees beugt zudem Ablagerungen wie Harngrieß und Harnsteinen in den Nieren, Harnleitern und der Harnblase vor.

Nach Informationen von Sidroga, Bad Ems

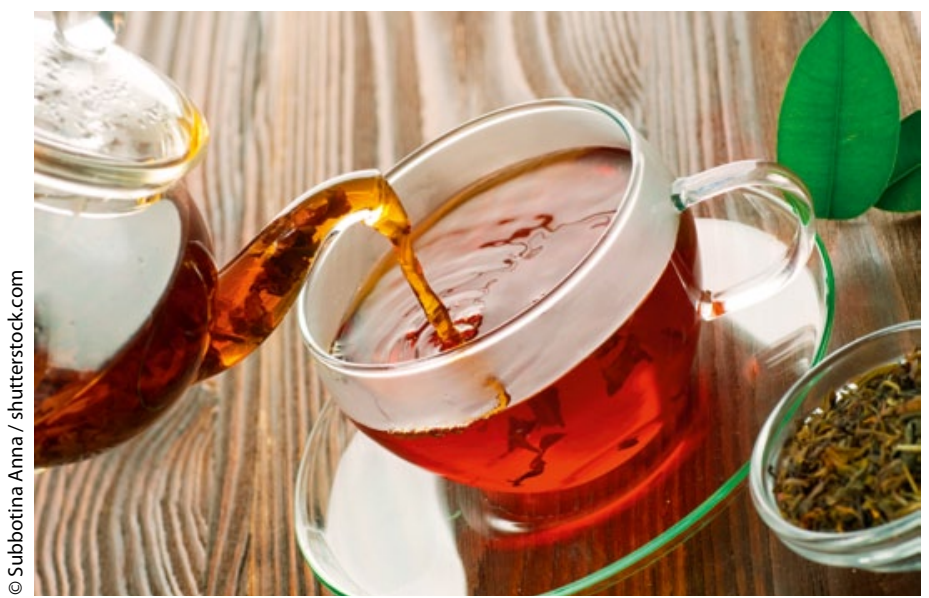

Heilpflanzentees eignen sich gut zum Ausspülen der Bakterien bei einer Blasenentzündung

\section{Weniger Zystoskopien mit} NMP22 in der Redizivkontrolle

— Die Zahl der Zystoskopien bei der Rezidivkontrolle von Urothelkarzinomen könnte mithilfe des Tumormarkers NMP22 deutlich gesenkt werden. Das ist das Ergebnis einer vorab in der elektronischen Ausgabe der Fachzeitschrift "Cancer" veröffentlichten Studie [Shariat SF et al. Cancer 2011].Wie die US-Wissenschaftler von der Cornell University, New York, zeigten, verbessert der Nachweis von NMP22 im Urin die Erkennung von Rezidiven des Urothelkarzinoms und auch von dessen Fortschreiten statistisch und klinisch signifikant. Voraussetzung: NMP22 fließt als zusätzlicher Parameter neben Kriterien wie Urinzytologie, Alter und Geschlecht in das Vorhersagemodell ein. Dank eines solchen Vorhersagemodells könnten Patienten, die aufgrund einer früheren, nicht muskelinvasiven Form von Blasenkrebs überwacht werden, viele Zystoskopien erspart werden. Bei ihnen könne so lange auf eine Zystoskopie verzichtet werden, bis sich der Verdacht auf Malignität erhärtet, so die Wissenschaftler. Die Berücksichtigung des Tumormarkers NMP22 steigert den Forschern zufolge die prädiktive Richtigkeit des Modells zur Vorhersage von Rezidiven um 20,8\%. Wenn es um das Fortschreiten der Erkrankung geht, beträgt dieser Wert sogar $43 \%$. Untersucht wurden insgesamt 2.222 Patienten mit einem histologisch bestätigtem Urothelkarzinom der Blase ohne Anzeichen einer Muskelinvasion (Stadien Ta, $\mathrm{T} 1$ und/oder Carcinoma in situ) und gleichzeitiger negativer Urinzytologie.

Nach Informationen von

Alere, Köln 